\title{
Comments on: Games with a permission structure - A survey on generalizations and applications
}

\section{A. Jiménez-Losada ${ }^{1}$}

Published online: 21 February 2017

(C) Sociedad de Estadística e Investigación Operativa 2017

In a cooperative situation the relationships among the agents may cause restrictions on coalition formation. As the author says, the best studied restrictions arise from communication and hierarchical relations. The analysis of cooperative games with a hierarchical structure in the players began with the study of permission structures (Gilles et al. 1992) and the introduction of games with structures of precedence (Faigle and Kern 1992). These papers marked two different options to deal with the problem of restricted cooperation by hierarchies. Over the past 25 years, permission systems have been developed, generalized and applied in different ways. Increasingly complex mathematical structures have been used for this. Hence, this survey about the subject is appropriate and convenient at this moment.

A hierarchical structure represents certain dependency relations among the agents. Different assumptions can be made about how these relationships restrict the cooperation among the players. In conjunctive permission structures (Gilles et al. 1992) the active participation of a player within a coalition requires the active participation of all their predecessors within the coalition. In the disjunctive approach (van den Brink 1997), the active participation of just one of the predecessors is required. An antimatroid (Algaba et al. 2004) shows these dependencies by the so called paths of the players, each $i$-path for a player $i$ represents a dependency relationship between player $i$ and the other players in this path. This is actually a partial dependency, because $i$ can use any $i$-path to participate in the game. Theorem 7 expresses how permission structures are extended by antimatroids. Technically, the analysis of games with permission structure is a nice mathematical problem, in the sense that the axiomatizations

This comment refers to the invited paper available at doi:10.1007/s11750-017-0440-9.

\footnotetext{
$凶 \quad$ A. Jiménez-Losada ajlosada@us.es

1 University of Seville, Seville, Spain
} 
of the values are always natural and reasonable, moreover, they allow to understand the intervention of the structure in the solution. Later on, local permission structures (van den Brink and Dietz 2014) separated the idea of authorization and participation. The three models showed in the paper represent different extensions of hierarchical structures. This survey makes clear the evolution of the model and it explains the mean results. But, for the sake of completeness, two more models should be considered as extensions of permission structures being hierarchical situations.

Each antimatroid $\mathcal{A}$ is identified with an interior operator int ${ }_{\mathcal{A}}$ (see in the paper). This interior operator determines, for each coalition, the largest active subcoalition into it taking into account the structure. But an interior operator does not necessarily represent an antimatroid. Derks and Peters (1993) introduced games with restrictions. Although the context of these games is not the analysis of hierarchical structures, restrictions are actually interior operators, as long as we admit that the interior of the grand coalition $i$ not necessarily the grand coalition. Restrictions can be considered as a generalization of permission structures. A restriction (interior operator) over $N$ is a mapping $\rho: 2^{N} \rightarrow 2^{N}$ satisfying for all set $E \subseteq N:(1) \rho(E) \subseteq E$, (2) $\rho(E) \subseteq \rho(F)$ if $E \subseteq F$, and (3) $\rho(\rho(E))=\rho(E)$. Derks and Peters (1993) considered a restricted game as $v^{\rho}(E)=v(\rho(E))$ for each game $v$ over $N$. But the axiomatization of the value for games with a restriction is not as natural and clear as the axiomatization of the values for games with permission structure or antimatroid. Moreover, restrictions were not explained as hierarchical structures.

Recently, Gallardo et al. (2015) have introduced a new extension of permission structures from interior operators: fuzzy authorization operators. A crisp authorization operator (a particular case of fuzzy authorization operator, see also Gallardo 2005) is a monotone mapping that determines a subcoalition for each coalition, namely $A: 2^{N} \rightarrow 2^{N}$ satisfying for each $E \subseteq N:(1) A(E) \subseteq E$ and (2) $A(E) \subseteq A(F)$ if $E \subseteq F$. Thus authorization operators differ from the interior operators in the property of idempotence. The restricted game is also defined as $v^{A}(E)=v(A(E))$. The authors defined partial dependency and veto power relationships in this new model. Therefore, authorization structures are useful for studying hierarchical situations. Authorization structures encompass local permission structures and restrictions. Obviously from now restrictions can be explained as hierarchical structures. Furthermore, the axiomatization of the value in Gallardo et al. (2015) is inspired by the axiomatization of the values for games with permission structure. An opening problem is how to discriminate restrictions into authorization structures by nature reasons.

Finally, I would like to highlight the effort of the author to compare communication and hierarchical relations. Future research can come from mixed models and their representation through mathematical structures.

\section{References}

Algaba E, Bilbao JM, Brink R, van den Jiménez-Losada A (2004) Cooperative games on antimatroids. Discrete Math 282:1-15

Derks J, Peters H (1993) A Shapley value for games with restricted coalitions. Int J Game Theory 21:351360 
Faigle U, Kern W (1992) The Shapley value for cooperative games under precedence constraints. Int J Game Theory 21:249-266

Gallardo JM (2015) Values for games with authorization structure. PhD Thesis. University of Seville, Spain Gallardo JM, Jiménez N, Jiménez-Losada A, Lebrón E (2015) Games with fuzzy authorization structure. Fuzzy Sets Syst 272:115-125

Gilles RP, Owen G, van den Brink R (1992) Games with permission structures: the conjunctive approach. Int J Game Theory 20:277-293

van den Brink R (1997) An axiomatization of the disjunctive permission value for games with a permission structure. Int J Game Theory 26:27-43

van den Brink R, Dietz C (2014) Games with local permission structure: separation of authority and value generation. Theory Decis 76:343-361 